\title{
ON THE OPTIMALITY OF SUBBAND ADAPTIVE FILTERS
}

\author{
Stephan Weiss and Robert W. Stewart \\ Department of Electronic and Electrical Engineering \\ University of Strathclyde, Glasgow G1 1XW, Scotland, UK \\ $\{$ weiss, bob\}@spd. eee.strath.ac.uk
}

\begin{abstract}
In this paper, we derive a polyphase analysis to determine the optimum filters in a subband adaptive filter (SAF) system. The structure of this optimum solution deviates from the standard SAF approach and presents its best possible solution only as an approximation. Besides this new insight into SAF error sources, the discussed analysis allows to calculate the optimum subband responses and the standard SAF approximation. Examples demonstrating the validity of our analysis and its use for determining SAF errors are presented.
\end{abstract}

\section{INTRODUC'TION}

Adaptive filtering in subbands is a useful approach to a number of problems such as acoustic echo cancellation $[1,2]$, identification of room acoustics [3], equalization of acoustics [4], or beamforming [5], where high computational cost can be reduced by processing in decimated subband signals. In Fig. 1, a subband adaptive filter (SAF) is shown in a system identification setup of an unknown system $s[n]$, whereby the input $x[n]$ and the desired signal $d[n]$ are split into $K$ frequency bands by analysis filter banks built of bandpass filters $h_{k}[n]$. Assuming a cross-band free SAF design

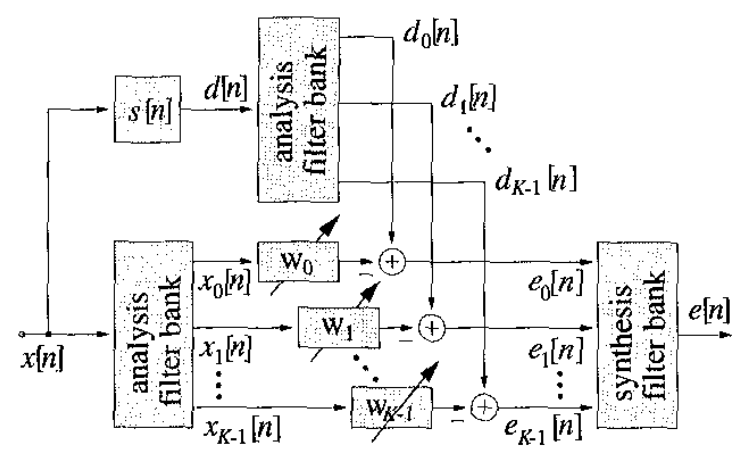

Figure 1: Subband adaptive filter (SAF) in system identification setup.

[2], an adaptive filter $w_{k}[n]$ is applied to each subband decimated by $N \leq K$. Finally, the fullband crror signal $e[n]$ can be reconstructed via a synthesis bank. The structures of both analysis and synthesis is shown in Fig. 2. Ideally, the overall system consisting of analysis and synthesis should only implement a delay, i.e. $\hat{x}[n]=x[n-\Delta]$.

However, subband adaptive filters (SAF) are subject to a number of limitations, which have been investigated, for example, with

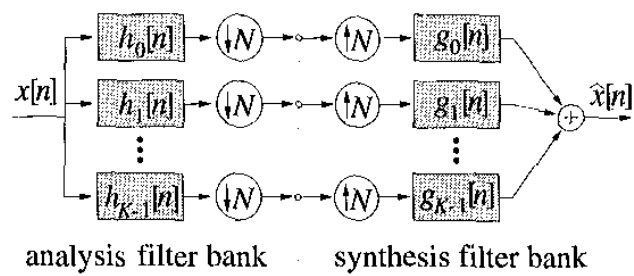

Figure 2: Analysis and synthesis filter bank performing a signal decomposition into $K$ frequency bands decimated by $N \leq K$.

respect to the required filter length $[2,6]$ or to lower bounds for the MMSE and the modelling accuracy [8]. These analyses have been performed using modulation description [2], time domain [6], or frequency domain approaches $[1,7,8]$.

Here, we discuss an SAF system as shown in Fig. 1 using a polyphase description [9] of its signals and filters. Sec. 2 reviews the idea of the polyphase expansion and presents the analysis of all involved signals. In Sec. 3, we introduce the formulation for the optimum subband adaptive filters, which will require a modification to the structure given in Fig. 1. We discuss in detail, how this optimum solution relates to the level of optimality, that is achicvable with the standard adaptive structure in Fig. 1, which will allow an assessment of the errors occurring in such standard SAF systems. Sec. 4 will discuss an example to highlight the use and insight reached by the analysis presented here.

\section{POLYPHASE ANALYSIS}

First, we derive expressions for the z-transforms for the decimated desired signal in the $k$ th subband, $D_{k}^{d}(z) \bullet \cdots d_{k}[n]$, and for the decimated input signal in the $k$ th subband, $X_{k}^{d}(z) \bullet x_{k}[n]$, as labelled in Fig. 1. This will allow us to assemble the z-transform of the $k$ th decimated subband error signal, $E_{k}^{d}(z) \bullet e_{k}(z)$. In our notation, superscript $\{.\}^{d}$ for z-transforms of signals refers to decimated quantities, while normal variables such as $X_{k}(z)$ indicate undecimated signals, i.e. in this case the input signal in the $k$ th subband before going into the decimator as shown in Fig. 2 .

\subsection{Polyphase Expansion}

The decimator and upsamplers in Fig. 2 are linear periodically time-varying (LPTV) operations, which makes it difficult to apply standard analysis tools for linear time-invariant (LTI) systems. However, polyphase analysis [10, 9] allows to express LPTV systems mostly as multiple-input multiple-output (MIMO) LTI sys- 
(a)

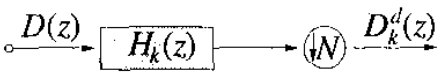

(b)

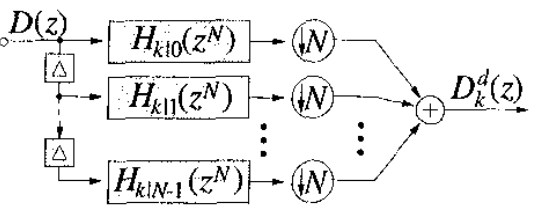

(c)

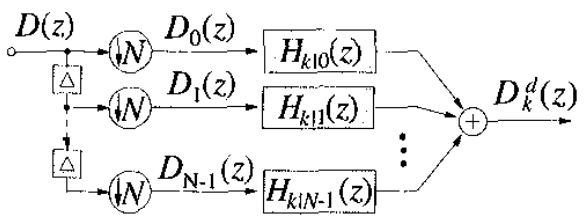

Figure 3: Analysis and synthesis fitter bank performing a signal decomposition into $K$ frequency bands decimated by $N \leq K$.

tems, with decimators and upsamplers being described by multiplexing and demultiplexing operations.

Considering the $\mathrm{z}$-transform of the $k$ th analysis filter, $H_{k}(z)$, $H_{k}(z) \bullet h_{k}[n]$, it can be written in expansion form

$$
H_{k}(z)=\sum_{n=0}^{N-1} z^{-n} \cdot H_{k, n}\left(z^{N}\right)
$$

where $H_{k, n}(z), n=0(1) N-1$, are the $N$ polyphase components of $H_{k}(z)$. Fig. 3 shows the effect of this expansion as applied in the desired path of the SAF structure (compare to Figs. 1 and 2). While Fig. 3(a) contains the $k$ th branch of the analysis operation applied to the desired signal, Fig. 3(b) represents the flow graph using the expansion (1). It is now possible to exploit the first Nobel identity [11] to swap the decimators with the polyphase filters $H_{k, n}\left(z^{N}\right)$ in Fig. 3(b), resulting in the structure shown in Fig. 3(c). Effectively, filtering now is performed at the lowest possible rate.

The multiplexed signals fed into the polyphase filters $H_{k, n}(z)$ are obtained by an analogous polyphase expansion of the desired signal $D(z)$,

$$
D(z)=\sum_{n=0}^{N-1} z^{-n} \cdot D_{n}\left(z^{N}\right) .
$$

Defining vector notation for the polyphase components of $H_{k}(z)$ and $D(z)$,

$$
\begin{aligned}
\underline{D}(z) & =\left[\begin{array}{llll}
D_{0}(z) & D_{1}(z) & \cdots & D_{N-1}(z)
\end{array}\right]^{T} \\
\underline{H}_{k}(z) & =\left[\begin{array}{llll}
H_{k \mid 0}(z) & H_{k \mid 1} & \cdots & H_{k \mid N-1}(z)
\end{array}\right]^{T^{\prime}}
\end{aligned}
$$

it is possible to express the $k$ th desired signal decimated by a factor $N$ as

$$
D_{k}^{d}(z)=\underline{H}_{k}^{T}(z) \cdot \underline{D}(z) .
$$

Note, that the mathematical expression (5) directly refers to the structure in Fig. 3(c).

\subsection{Description of Subband Desired Signal}

Further to the analysis in Sec. 2.1, we want to trace the decimated desired subband signal $D_{k}^{d}(z)$ back to the input signal, $X(z) \bullet$ $x[n]$. Through the unknown system in Fig. 1, the relation between input and desired signal is given by $D(z)=S(z) \cdot X(z)$, where $S(z) \bullet s[n]$ is the z-transform of the unknown system. With some effort, this expression for the desired signal can be appropriately expanded such that the $n$th polyphase component in (3) is given by

$$
D_{n}(z)=\underline{S}^{T}(z) \cdot \Lambda_{n}(z) \cdot \underline{X}(z)
$$

The polyphase vectors $S(z)$ and $X(z)$ refer to the unknown system $S(z)$ and the input signal $X \overline{(z)}$ in analogy to the definitions (3) and (2). The matrix $\boldsymbol{\Lambda}_{n}(z)$ is a delay matrix defined as

$$
\boldsymbol{\Lambda}_{n}(z)=\left[\begin{array}{cc}
\mathbf{0} & \mathbf{I}_{N-n} \\
z^{-1} \mathbf{I}_{n} & \mathbf{0}
\end{array}\right]
$$

Thus, the overall description for the decimated $k$ th desired subband signal yields

$$
D_{k}^{d}(z)=\underline{H}_{k}^{T}(z)\left[\begin{array}{c}
\underline{S}^{T}(z) \boldsymbol{\Lambda}_{0}(z) \\
\underline{S}^{\prime}(z) \boldsymbol{\Lambda}_{1}(z) \\
\vdots \\
\underline{S}^{T}(z) \boldsymbol{\Lambda}_{N-1}(z)
\end{array}\right] \underline{X}(z)=\underline{H}_{k}^{T}(z) \mathbf{S}(z) \underline{X}(z)
$$

where the symmetric matrix $\mathbf{S}(z)=\mathbf{S}^{T}(z)$ has been substituted for brevity. Now the unknown system has been swapped with the multiplexing operation in Fig. 3(c).

\subsection{Description of Subband Input and Error Signals}

Similarly to the previous analysis, the $k$ th decimated input signal can be derived as

$$
X_{k}^{d}(z)=\underline{H}_{k}^{T}(z) \cdot \underline{X}(z)
$$

Finally, we use (8) and (9) to formulate the $k$ th subband error signal, $E_{k}^{d}(z) \bullet-e_{k}[n]$, including the $k$ th adaptive filter with ztransform $W_{k}(z) \bullet \circ w_{k}[n]$ :

$$
\begin{aligned}
E_{k}^{d}(z) & =D_{k}^{d}(z)-W_{k}(z) \cdot X_{k}^{d}(z) \\
& =\left\{\underline{H}_{k}^{T^{\prime}}(z) \cdot \mathbf{S}(z)-\underline{H}_{k}^{T}(z) \cdot W_{k}(z)\right\} \underline{X}(z) .
\end{aligned}
$$

Hence, polyphase descriptions for all involved decimated subband signals have been derived. In particular, note that the desired subband signal now is entirely expressed in terms of the polyphase components of both the analysis filters, the unknown system, and the input signal.

\section{OPTIMUM SUBBAND FILTERS}

In the following, we use the expressions found in Sec. 2 to obtain an optimum solution for the adaptive subband filters, $W_{k}(z)$. 


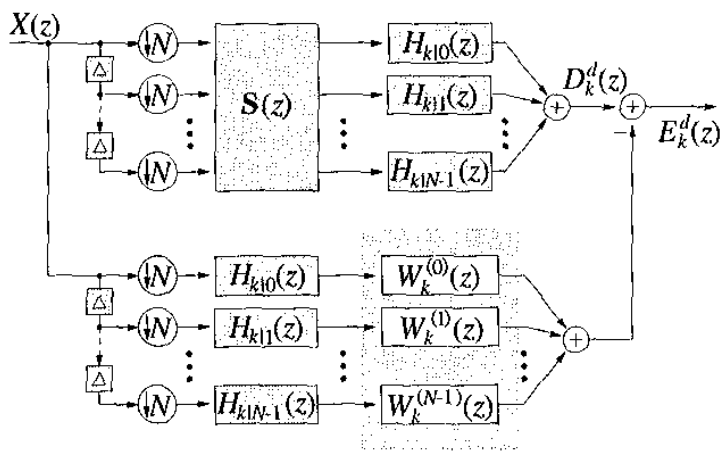

Figure 4: SAF optimal polyphase solution in the $k$ th subband.

\subsection{Error Minimization}

Assuming that no disturbances are present and the SAF system in Fig. 1 can perfectly model the unknown system, $E_{k}^{d}(z)$ should be zero in the steady statc. As it is desirable to achicve optimality of the subband filters regardless of the input, the requirement for optimality (in every sense) is

$$
\underline{H}_{k}^{T}(z) \cdot \mathbf{S}(z) \stackrel{!}{=} \underline{H}_{k}^{T} \cdot W_{k, \text { opt }}(z)
$$

following from (11). Hence, we obtain $N$ cancellation conditions indicated by superseripts $\{\cdot\}^{(n)}$, which have to be fulfilled:

$$
W_{k, \mathrm{opt}}^{(n)}(z)=\frac{\underline{H}_{k}^{T}(z) \cdot \Lambda_{n}^{T}(z) \cdot \underline{S}(z)}{H_{k \mid n}(z)} \quad \forall n \in\{0 ; N-1\} .
$$

Therefore, ideally $W_{k}(z)$ in (11) and (12) should be replaced by an $N \times N$ diagonal matrix with entries $W_{k}^{(n)}(z)$. For the $k$ th subband, this solution with $N$ polyphase filters is given by the structure in Fig. 4.

\subsection{Discussion}

An alternative notation to (13) is to write the $n$th optimum solution as

$$
W_{k, \mathrm{opt}}^{(n)}(z)=\sum_{\nu=0}^{N-1} A_{k \mid n}^{(\nu)}(z) \cdot S_{\nu}(z)
$$

and interpret it as a superposition of polyphase components of $S(z)$, "weighted" by transfer functions

$$
A_{k \mid n}^{(\nu)}(z)=z^{-\lfloor(n+\nu) / N\rfloor} \cdot \frac{H_{k \mid(n+\nu) \bmod _{N}}(z)}{H_{k \mid n}(z)}
$$

This forms the basis for some interesting observations,

Firstly, the length of the optimum subband responses is obviously given by $1 / N$ of the order of $S(z)$, but extended by the transfer functions (15). These extending transients are causal for poles of $A_{k \mid n}^{(\nu)}(z)$ within the unit circle, and non-causal for stabilized poles outside the unit-circle [12]. Hence, besides the motivation for a non-causal optimum response, it is particularly interesting that the required SAF length obviously depends on the transients caused by the analysis filters $H_{k}(z)$.

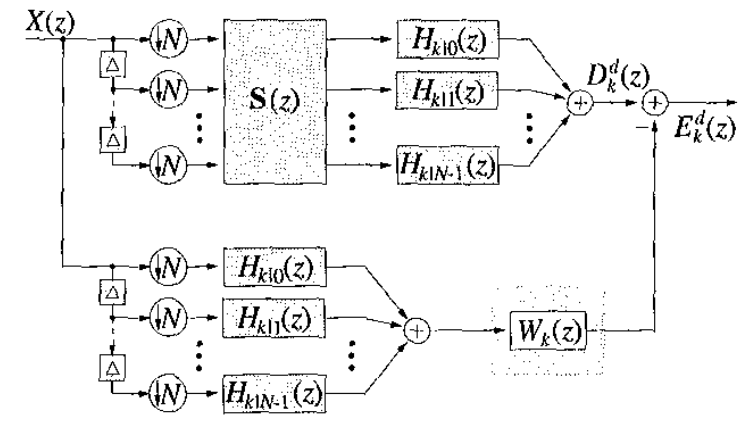

Figure 5: SAF standard solution in the $k$ th subband.

Secondly in general, particularly when the stopband attenuation of the analysis filters is insufficient, the components $H_{k \mid n}(z)$ in (15) differ, hence leading to different polyphase solutions $W_{k, \text { opt }}^{(n)}(z)$ in every of the $N$ branches in Fig. 4. Only if all elements in (15) are identical, the optimal subband responses can be swapped with the adder and give the well-known standard SAF solution shown in Fig. 5.

Thus, if non-ideal filter banks are used and in particular aliasing is present in the subband signals, this optimum standard SAF solution gives the closest $l_{2}$ match to all $N$ optimal polyphase solutions:

$$
W_{k, \mathrm{opt}}(z)=\frac{1}{N} \sum_{n=0}^{N-\mathrm{i}} W_{k, \mathrm{opt}}^{(n)}(z)
$$

The error made in this approximation can explain MMSE and modelling limitations of the SAF approach and represents an alternative coefficient / time-domain description as opposed to spectrally motivated SAF error explanations in the literature $[2,8]$.

\section{EXAMPLES}

To verify the validity of our analysis, we first discuss an unrealistic, but very simple example of a critically decimated 2-channel SAF system using Haar filters [9]. We want to identify the unknown system $S(z)=1+z^{-1}$ using a unit variance Gaussian white noise excitation, and here only consider the lowpass band produced by the analysis Haar filter $H_{0}(z)=1+z^{-1}$. Evaluating (14) and (15) yields as optimum polyphase solution

$$
W_{0, \mathrm{opt}}^{(0)}(z)=2, \quad W_{0, \mathrm{opt}}^{(1)}(z)=1+z^{-1} .
$$

In a simulation using a recursive least squares (RLS) algorithm [13], the converged adaptive filter $W_{0}(z)=1.4873+0.5067 z^{-1}$ very closely agrees with the analytical solution (16) calculated from (17),

$$
W_{0, \mathrm{opt}}(z)=1.5+0.5 z^{-1}
$$

Additionally, the PSD of the 0th adapted subband error signal, $S_{e_{0}}\left(e^{j \Omega}\right)$, can be analytically predicted by inserting the optimum standard solution (16) into (11),

$$
S_{e_{0}}\left(e^{j \Omega}\right)=\left|E_{k}^{i}\left(e^{j \Omega}\right)\right|^{2}=1-\cos \Omega
$$




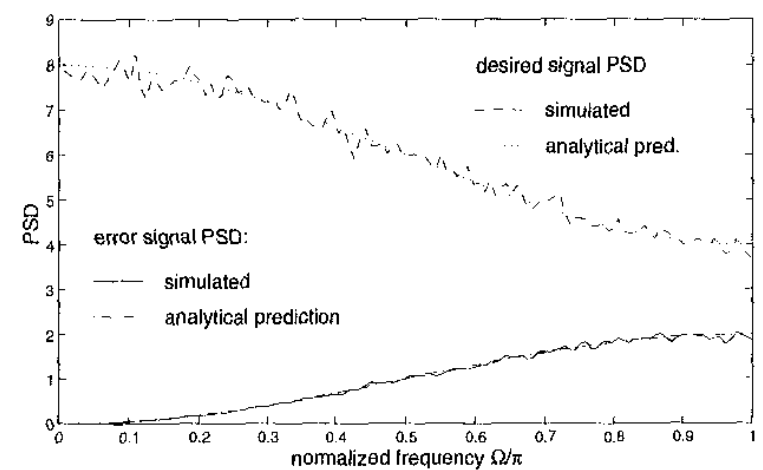

Figure 6: Comparison between simulated and analytically predicted PSDs in the 0th subband.

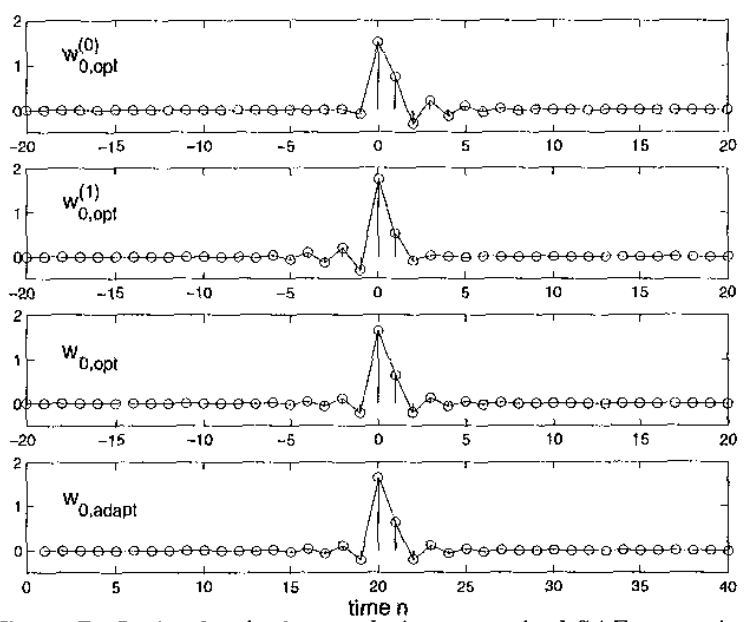

Figure 7: Optimal polyphase solutions, standard SAF approximation, and simulation result of a system using a 32 tap analysis filter.

which can be used to determine the minimum mean squared error of the SAF system alternative to spectral methods [8]. Fig. 6 demonstrates the excellent fit between the analytically calculated PSD in (18), and the measured results from the RLS simulation. Also shown is the analytically predicted and measured PSD of the 0th desired subband signal $S_{d_{0}}\left(e^{j \Omega}\right)=6+2 \cos \Omega$ (hence the uncancelled error signal) calculated via (5).

As a second example, Fig. 7 shows analytical and simulated results for the 0th subband in critically sampled 2-channel SAF system employing the 32 tap QMF filter 32C [14]. The responses $w_{0, \mathrm{opt}}^{(0)}[n]$ and $w_{0, \mathrm{opt}}^{(1)}[n]$ are the optimum polyphase solutions as indicated in Fig. 4. In the two bottom diagrams, the analytical solution (16) for the best approximation $w_{k, \text { opt }}[n]$ of the standard SAF setup in Fig. 5 closely agrees to the result of an RLS solution, $w_{0, \text { adapt }}[n]$.

\section{CONCLUSION}

We have introduced a polyphase analysis of an SAF system, which leads to an optimum polyphase solution for the subband filters, which can be computed using the formulations presented here. Interestingly, the standard SAF solution can only allow an approx- imation of these optimal polyphase solutions, which gives alternative insight into the inaccuracies and limitations of the SAF approach. Thus, the 'classical' error explanation by aliasing $[1,7,8]$ is replaced by the approximation of potentially differing polyphase solutions. Therefore potential of the presented analysis lies in the access to the optimum and approximate solutions, which may complement analysis with regard to other error sources [8].

\section{ACKNOWLEDGMENT}

The authors would like to gratefully acknowledge Dr. Ian Proudler of DERA, Malvern, England, who partially supported this work.

\section{REFERENCES}

[1] W. Kellermann, "Analysis and Design of Multirate Systems for Cancellation of Acoustical Echoes", in Proc. IEEE ICASSP, New York, 1988, vol. 5, pp. 2570-2573.

[2] A. Gilloire and M. Vetterli, "Adaptive Filtering in Subbands with Critical Sampling: Analysis, Experiments and Appljcations to Acoustic Echo Cancelation", IEEE Trans SP, vol. SP-40, no. 8, pp. 1862-1875, Aug. 1992.

[3] M. Schönle, N.J. Fliegc, and U. Zölzer, "Parametric Approximation of Room Impulse Responses by Multirate Systems", in Proc. IEEE ICASSP, Minneapolis, 1993, vol. I, pp. 153156.

[4] S. Weiss, S.R. Dooley, R.W. Stewart, and A.K. Nandi, "Adaptive Equalization in Oversampled Subbands", IEE Electr. Let., vol. 34, no. 15, pp. 1452-1453, July 1998.

[5] W. Kellermann, "Strategies for Combining Acoustic Echo Cancellation and Adaptive Beamforming Microphone Arrays", in Proc. IEEE ICASSP, Munich, 1997, vol. I, pp. 219222.

[6] R.J. Wilson, P.A. Naylor, and D.M. Brookes, "Performance Limitations of Subband Acoustic Echo Controllers", in Proc. IWAENC, Impcrial College, London, 1997, pp. 176179.

[7] M.R. Petraglia and S.K. Mitra, "Performance Analysis of Adaptive Filter Structures Based on Subband Decompositions", in Proc. ISCAS, Chicago, 1993, vol. I, pp. 60-63.

[8] S. Weiss, R.W. Stewart, A. Stenger, and R. Rabenstein, "Performance Limitations of Subband Adaptive Filters", in Proc. EUSIPCO, Rodos, Greece, 1998, vol. III, pp. 12451248.

[9] N.J. Fliege, Multirate Digital Signal Processing: Multirate Systems, Filter Banks, Wavelets, John Wiley \& Sons, Chichester, 1994.

[10] P. Vary, "On the Design of Digital Filter Banks Based on a Modified Principle of Polyphase", Archiv Elektr. Übertr., vol. 33, pp. 293-300, 1979.

[11] P.P. Vaidyanathan, Multirate Systems and Filter Banks, Prentice Hall, Englewood Cliffs, 1993.

[12] B. Widrow and E. Walach, Adaptive Inverse Control, Prentice Hall, Englewood Cliffs, 1995.

[13] S. Haykin, Adaptive Filter Theory, Prentice Hall, Englewood Cliffs, 2nd ed. , 1991.

[14] R.E. Crochiere and L.R. Rabiner, Multirate Digital Signal Processing, Prentice Hall, Englewood Cliffs, 1983. 